\title{
Systematic reviews on interventions for COVID-19 have rarely graded the certainty of the evidence
}

\author{
Ana Luiza Cabrera Martimbianco', Rafael Leite Pacheco", Carolina de Oliveira Cruz Latorraca"', Raphael Einsfeld Simões Ferreira"v, \\ Rachel Rierav
}

Centro Universitário São Camilo, São Paulo (SP), Brazil

'PhD. Professor, Centro Universitário São Camilo, São Paulo (SP), Brazil; and Professor, Universidade Metropolitana de Santos (UNIMES), Santos (SP), Brazil.

(D) https://orcid.org/0000-0002-4361-4526.

"MSc. Professor, Centro Universitário São Camilo, São Paulo (SP), Brazil; and Researcher, Escola Paulista de Medicina, Universidade Federal de São Paulo (EPM-UNIFESP), São Paulo (SP), Brazil.

(D) https://orcid/org/0000-0001-7487-8471

'"MSc. Researcher, Escola Paulista de Medicina, Universidade Federal de São Paulo (EPM-

UNIFESP), São Paulo (SP), Brazil.

(D) https://orcid/org/0000-0001-9146-4684

"vphD. Professor, Centro Universitário São Camilo, São Paulo (SP), Brazil.

(D) https://orcid/org/0000-0002-1167-142X

vPhD. Adjunct Professor, Discipline of EvidenceBased Health, Escola Paulista de Medicina, Universidade Federal de São Paulo (EPMUNIFESP), São Paulo (SP), Brazil; and Coordinator, Health Technology Assessment Center, Hospital Sírio-Libanês, São Paulo (SP), Brazil.

(D) https://orcid/org/0000-0002-9522-1871

KEY WORDS (MeSH terms):

COVID-19.

Systematic review [publication type].

Evidence-based practice.

GRADE approach.

Evidence-based medicine.

Public health.

AUTHORS' KEY WORDS:

Methodology.

Certainty of evidence.

Strength of recommendations.

Evidence-based healthcare.

\begin{abstract}
BACKGROUND: Numerous systematic reviews on coronavirus disease-19 (COVID-19) treatment have been developed to provide syntheses of the large volume of primary studies. However, the methodological quality of most of these reviews is questionable and the results provided may therefore present bias. OBJECTIVE: To investigate how many systematic reviews on the therapeutic or preventive options for COVID-19 assessed the certainty of the evidence through the Grading of Recommendations Assessment, Development and Evaluation (GRADE) approach.

METHODS: We conducted a sensitive search in MEDLINE (via PubMed) and included all systematic reviews that assessed any intervention for COVID-19. The systematic reviews included were examined to identify any planned and/or actual assessment using the GRADE approach (or absence thereof) regarding the certainty of the evidence.

RESULTS: We included 177 systematic reviews and found that only 37 (21\%; 37/177) assessed and reported the certainty of the evidence using the GRADE approach. This number reduced to 27 (16.2\%; 27/167) when Cochrane reviews ( $n=10$ ), in which an evaluation using GRADE is mandatory, were excluded.

CONCLUSION: Most of the systematic reviews on interventions relating to COVID-19 omitted assessment of the certainty of the evidence. This is a critical methodological omission that must not be overlooked in further research, so as to improve the impact and usefulness of syntheses relating to COVID-19.
\end{abstract}

\section{INTRODUCTION}

Since the beginning of the coronavirus disease 2019 (COVID-19) pandemic, large numbers of studies have been published in an attempt to find an effective treatment for this disease. Consequently, many systematic reviews have been developed on this topic, to provide syntheses of the large volume of primary studies. Healthcare professionals and policymakers commonly use systematic reviews to formulate recommendations and make practical decisions. ${ }^{1}$

However, the methodological quality of most of these systematic reviews is questionable. Hence, the results provided through these reviews may present bias.

Assessing the certainty of the evidence is an indispensable step in a systematic review. This is especially true within the current context, in which information is often misleading yet has been widely disseminated, both by scientific journals and by the traditional media. Thus, efforts need to be made by the authors of syntheses of the evidence on a given topic, to ensure that the degree of certainty that can be placed on the estimates of effect and clinical recommendations can be established. ${ }^{1,2}$

The Grading of Recommendations Assessment, Development and Evaluation (GRADE) is a transparent approach to rating the certainty of the body of evidence in systematic reviews and other forms of synthesis, as a guide to making decisions. ${ }^{3}$ This approach should be beneficial during the COVID-19 pandemic. It is entirely possible to perform GRADE assessments, even (or even more so) within an emergency context. ${ }^{1,4}$ 


\section{OBJECTIVE}

We carried out a critical appraisal study with the aim of investigating how many systematic reviews that have been published in relation to therapeutic or preventive options for COVID-19 made assessments of the certainty of the evidence through the GRADE approach.

\section{METHODS}

We conducted a sensitive search in MEDLINE (via PubMed) on January 20, 2021, using the MeSH term "Coronavirus" and its synonyms combined with the PubMed clinical queries filter for systematic reviews (Annex 1). Two authors independently screened all titles and abstracts through the Rayyan platform, ${ }^{5}$ in order to include systematic reviews that assessed any intervention for COVID-19.

\section{RESULTS}

The systematic reviews included were analyzed in full text, to identify whether there was any planned and/or actual assessment of the certainty of the evidence using the GRADE approach (or absence thereof). The search strategy found 1,075 references, and 177 fulfilled the inclusion criteria. Of these, only 37 reviews $(21 \% ; 37 / 177)$ assessed the certainty of the evidence using the GRADE approach. This number reduced to 27 (16.2\%; 27/167) when Cochrane reviews $(\mathrm{n}=10)$, in which an evaluation using GRADE is mandatory, were excluded.

\section{CONCLUSION}

This result highlights the fact that most of the systematic reviews on interventions conducted in relation to COVID-19 omitted assessment of the certainty of the evidence. This is a critical methodological omission that must not be overlooked in further research, so as to improve the impact and usefulness of syntheses relating to COVID-19.

\section{REFERENCES}

1. Schünemann HJ, Santesso N, Vist GE, et al. Using GRADE in situations of emergencies and urgencies: certainty in evidence and recommendations matters during the COVID-19 pandemic, now more than ever and no matter what. J Clin Epidemiol. 2020:127:202-7. PMID: 32512187; http:// doi.org/10.1016/j.jclinepi.2020.05.030.

2. Kumar A, Taggarsi M. GRADEing quality of evidence and its importance in evidence-based practice. BMJ Evid Based Med. 2020:bmjebm-2020-111525. PMID: 33004427; http://doi.org/10.1136/ bmjebm-2020-111525.

3. Guyatt $G$, Oxman AD, Akl EA, et al. GRADE guidelines: 1. IntroductionGRADE evidence profiles and summary of findings tables. J Clin Epidemiol. 2011;64(4):383-94. PMID:21195583; http://doi.org/10.1016/j. jclinepi.2010.04.026.
4. Munn Z, Twaddle S, Service D, et al. Developing guidelines before, during, and after the COVID-19 pandemic. Ann Intern Med. 2020:173(12):1012-4. PMID: 32931327; http://doi.org/10.7326/M20-4907.

5. Ouzzani M, Hammady H, Fedorowicz Z, Elmagarmid A. Rayyan-a web and mobile app for systematic reviews. Syst Rev. 2016;5(1):210. PMID: 27919275; http://doi.org/10.1186/s13643-016-0384-4.

Authors' contributions: Martimbianco ALC: conceptualization (equal), data curation (equal), validation (equal), writing-original draft (equal) and writing-review and editing (equal); Pacheco RL: conceptualization (equal), data curation (equal), formal analysis (equal), validation (equal), visualization (equal), writing-original draft (equal) and writing-review and editing (equal); Riera R: conceptualization (equal), validation (equal), visualization (equal), writing-original draft (equal) and writing-review and editing (equal); Latorraca COC: formal analysis (equal), validation (equal), visualization (equal) writing-original draft (equal) and writing-review and editing (equal); and Ferreira R: formal analysis (equal), validation (equal), visualization (equal), writing-original draft (equal) and writing-review and editing (equal). All authors approved the final manuscript

Sources of funding: This research did not receive any specific grant from any funding agency in the public, commercial or not-for-profit sectors Conflict of interest: The authors declare that they did not have any competing interest

Date of first submission: February 8, 2021

Last received: February 8, 2021

Accepted: May 27, 2021

\section{Address for correspondence:}

Rafael Leite Pacheco

Centro Universitário São Camilo

Av. Nazaré, 1.501

Ipiranga — São Paulo (SP) — Brasil

CEP 04262-200

Tel. (+55 11) 3465-2664

E-mail: rleitepacheco@hotmail.com 
Annex 1. Search strategy.

Database

\section{Search strategy}

\#1 “Coronavirus"[Mesh] OR “Covid-19" OR (COVID) OR (Coronavirus) OR (SARS-CoV-2) OR (Coronaviruses) OR (Deltacoronavirus) OR (Deltacoronaviruses) OR "Munia coronavirus HKU13" OR (Coronavirus HKU15) OR (Coronavirus, Rabbit) OR (Rabbit Coronavirus) OR (Coronaviruses, Rabbit) OR (Rabbit Coronaviruses) OR "Bulbul coronavirus HKU11" OR "Thrush coronavirus HKU12"

MEDLINE \#2 (((systematic review[ti] OR systematic literature review[ti] OR systematic scoping review[ti] OR systematic narrative review[ti]

(via PubMed) OR systematic qualitative review[ti] OR systematic evidence review[ti] OR systematic quantitative review[ti] OR systematic metareview[ti] OR systematic critical review[ti] OR systematic mixed studies review[ti] OR systematic mapping review[ti] OR systematic cochrane review[ti] OR systematic search and review[ti] OR systematic integrative review[ti]) NOT comment[pt] NOT (protocol[ti] OR protocols[ti])) NOT MEDLINE [subset]) OR (Cochrane Database Syst Rev[ta] AND review[pt]) OR systematic review[pt] 